\title{
Photorefractive Wave Mixing in Periodically Poled $\mathrm{LiNbO}_{3}: \mathrm{Y}: \mathrm{Fe}$
}

\author{
S. Odoulov ${ }^{a}$, A. Shumelyuk ${ }^{a}$, O. Shinkarenko ${ }^{a}$, \\ D. BArilov ${ }^{a}$, S. PAVluUK ${ }^{a}$ AND B. Sturman ${ }^{b}$ \\ ${ }^{a}$ Institute of Physics, National Academy of Sciences \\ 03650, Kiev-39, Ukraine \\ ${ }^{b}$ International Institute for Nonlinear Studies \\ Koptyug Ave. 1, 630090, Novosibirsk, Russia
}

\begin{abstract}
The review of recent experimental work on photorefractive grating recording and frequency degenerate nonlinear wave mixing in periodically poled lithium niobate is given. We report, in particular, on new results concerning photorefractive grating recording in periodically poled lithium niobate by two orthogonally polarized eigenwaves (ordinary and extraordinary), on coherent self-oscillation in semilinear cavity, on evaluation of the effective photovoltaic field in iron-doped periodically poled lithium niobate.
\end{abstract}

PACS numbers: 42.65.Hw, 42.40.Pa, 77.80.-e

\section{Introduction}

Traditionally, periodically poled ferroelectric crystals are used to improve phase matching in different three-wave mixing processes [1-3] (sum and difference frequency generation, frequency doubling, optical parametric oscillation, etc.). We report on experimental study of different kinds of four-wave mixing in periodically poled lithium niobate (PPLN) deliberately doped with Y (to achieve periodic poling) and $\mathrm{Fe}$ (to enhance photorefractive response). The advantage of PPLN:Y:Fe consists in strongly inhibited nonlinear response at low spatial frequencies (i.e., reduced "optical damage") with strong response at high spatial frequencies, which allows for efficient grating recording [4-6].

\section{Physical background}

The nonlinearity of photorefractive crystals is due to the development of the space-charge field $E_{\mathrm{sc}}$ that modifies high-frequency dielectric constant via linear 
electrooptic effect (the Pockels effect) [7-9]. The light-induced refractive index change is given by

$$
\Delta n=-(1 / 2) n^{3} r_{\mathrm{eff}} E_{\mathrm{sc}},
$$

where $n$ is the refractive index and $r_{\text {eff }}$ is the effective electrooptic constant. The space-charge field may arise because of photoexcited carrier diffusion or charge redistribution in external electric field $E_{0}$. The ultimate value of the space-charge field $E_{\mathrm{sc}}$ is limited in this two cases by the diffusion field $E_{\mathrm{D}}=(2 \pi / \Lambda)\left(k_{\mathrm{B}} T / e\right)$ (where $k_{\mathrm{B}}$ is the Boltzmann constant, $T$ is the temperature, $e$ is the electron charge, and $A$ is the characteristic scale of the spatial intensity distribution, e.g., $\Lambda=\lambda /(2 \sin \theta)$ is the fringe spacing for grating recording, $2 \theta$ being the full angle between recording waves in air) or by applied electric field $E_{0}$.

In doped crystals with low conductivity an efficient charge transport process is the bulk photovoltaic effect [8], it may result in development of very large space charge fields up to $10^{4}-10^{5} \mathrm{~V} / \mathrm{cm}$. The steady state electric current is generated in the short-circuited illuminated sample, which is proportional in the simplest scalar case to the light intensity $I$ :

$$
j=\beta_{\text {eff }} I,
$$

where $\beta_{\text {eff }}$ is the effective photovoltaic constant. The amplitude of the space-charge field which is due to photovoltaic charge separation is equal to the effective photovoltaic field

$$
E_{\mathrm{pv}}=j / \sigma,
$$

where $\sigma$ is the crystal conductivity [8]

The refractive index change for photovoltaic photorefractive material is

$$
\Delta n=-(1 / 2) n^{3} r_{\text {eff }} \beta_{\text {eff }} \mathrm{I} / \sigma \text {. }
$$

The effect of the domain inversion on photorefractive grating recording depends on particular charge transport process. For diffusion-mediated or drift-mediated charge separation the sign of the space charge field is independent of orientation of the spontaneous polarization axis $P_{\mathrm{s}}$ and remains the same in adjacent domains while the effective electrooptic constant in Eq. (1) does depend on the sign of $P_{\mathrm{s}}$. This means that the contrast of the phase grating recorded in the sample is reversed in every new domain with inverted $P_{\mathrm{s}}$ direction. In other words there is a $\pi$-shift in phase of the recorded phase grating in every new domain. The total recorded structure looks like a sequence of thin stripes with complimentary phase gratings: maxima changed to minima and vice versa in adjacent domains, Fig. 1a. The partial contributions to the wave diffracted from the gratings at the Bragg angle in two adjacent domains will therefore be also out of phase and integration over total sample thickness will give a vanishing to zero intensity for the diffracted wave.

The result differs substantially in case of photovoltaic charge separation. Here the direction of photovoltaic current also depends on orientation of ferroelectric axis. If the axis of spontaneous polarization is inverted both the effective 


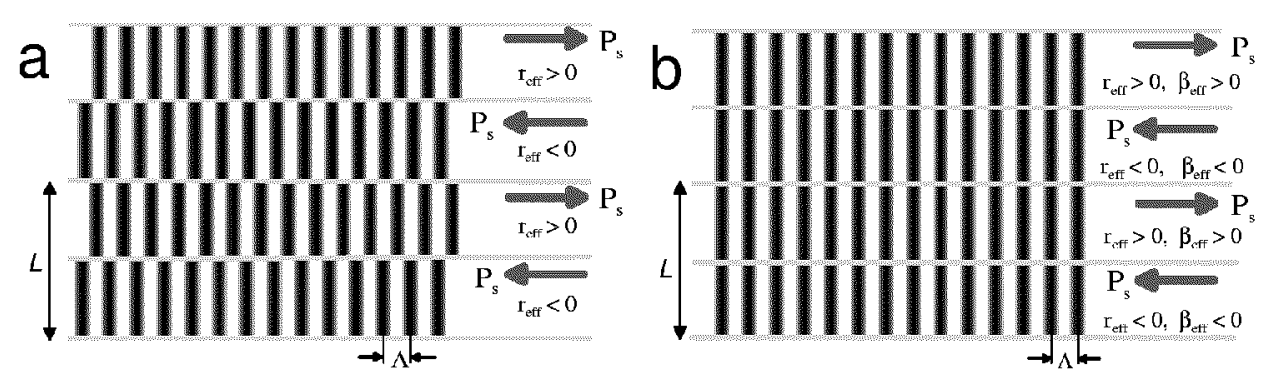

Fig. 1. Schematic representation of the refractive index grating structure in periodically poled lithium niobate with the diffusion driven charge transport (a) and with photovoltaic charge transport (b). $L$ is the domain lattice period and $A$ is the index grating spacing.

electrooptic constant $r_{\text {eff }}$ and effective photovoltaic constant $\beta_{\text {eff }}$ change their signs so that the sign of refractive index change remains unchanged (see Eq. (4)). In such a way there is no phase shift between photorefractive gratings recorded in domains with opposite direction of ferroelectric axis, Fig. 1b. The question remains, how strongly the amplitude of photorefractive grating is affected by the domain inversion.

The analytical solution for the spatial structure of the space-charge field in periodically doped crystals with photovoltaic charge transport was first derived in [10]. The main conclusion that can be extracted from this solution is that for light fringes with spacing much smaller than the domain lattice period $L$ the amplitude of the space charge field in PPLN sample approaches that in a perfect single-domain sample. If, on the contrary, the spatial variation of the light intensity becomes much larger than the typical domain width the corresponding space-charge field is decreasing. It vanishes to zero if the domain lattice is perfectly symmetric, i.e., the width of domain with positive $P_{\mathrm{s}}$ is equal to that of domain with negative $P_{\mathrm{s}}$.

The qualitative explanation of this behaviour is as follows. If the domain lattice period is much larger than the fringe spacing the space-charge field inside each domain approaches the field that would be induced in a single-domain crystal with the same orientation. The influence of the charges in other domains on the space-charge field in considered separate domain is relatively small. In adjacent domains with reversed $P_{\mathrm{s}}$ the space-charge field has the same strength and opposite direction. A strong variation of the space-charge field occurs only near the domain walls where the field $E_{\mathrm{sc}}$ passes a zero value. Until the thickness of the transitional layers between two antiparallel domains is small compared to the domain width $L / 2$ the space charge field in any particular domain is close to the effective photovoltaic field.

When the fringe spacing becomes larger the effect of the transitional layers becomes important and the space charge field starts to decrease. For $A \gg L$ (low 
spatial frequency $K=2 \pi / \Lambda$ ) the space charge field becomes strongly reduced because of a quick alternation of positive and negative photoinduced charges along each fringe. The calculated space-charge field component along the grating vector $\boldsymbol{K}$ is $[4,10]$ :

$$
E_{\mathbb{K}}=-E_{\mathrm{pv}}[1-(2 G / K) \tanh (K / 2 G)]
$$

where $G=2 \pi / L$ is the spatial frequency of the domain lattice. The normalized space charge field $E_{K} / E_{\mathrm{pv}}$ is plotted in Fig. 2 as a function of the normalized spatial frequency $(K / G)$.

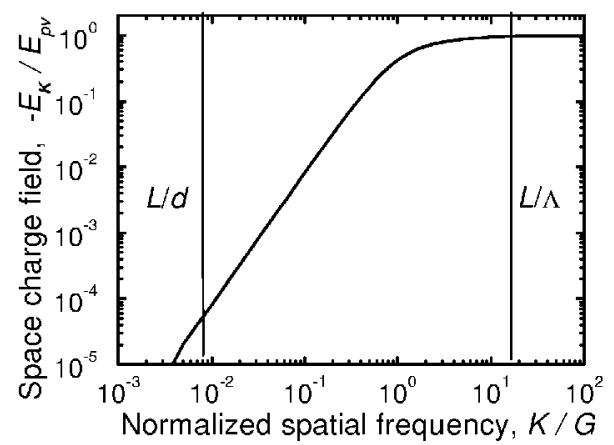

Fig. 2. Calculated dependence of photorefractive response versus spatial frequency of light intensity inhomogeneity. Two vertical lines show typical $K$, one (right) for the recording of photorefractive grating with $1 \mu \mathrm{m}$ spacing, the other one (left) for single laser beam with the beam waist $1.5 \mathrm{~mm}$. The PPLN domain lattice period about $7 \mu \mathrm{m}$ is assumed in calculations.

For small fringe spacing (large $K / G$ ) the space charge field $E_{K}$ approaches its ultimate value $E_{\mathrm{pv}}$ that can be reached in single domain crystal while for large scale intensity variation $(K \ll G) E_{\mathbb{K}}$ is decreasing as $E_{K} \propto(K / G)^{2}[4,10]$.

Thus the main advantage of doped photorefractive PPLN crystals as nonlinear medium for the grating recording and frequency degenerate four-wave mixing is its unusual spatial frequency response, with good sensitivity at high spatial frequencies (grating recording with high diffraction efficiency is possible) and suppressed sensitivity at low spatial frequencies (inhibited optical damage). We will present in what follows few examples how these properties are manifested in different types of the four-wave mixing in PPLN.

\section{Reduction of optical damage}

The first experimental demonstration of the optical damage inhibition was reported in our paper [5] for bulk PPLN crystal grown in Moscow State University from congruently melted composition by Czochralski technique [11], co-doped with 
$\mathrm{Y}$ and Fe. The iron content in PPLN samples used in these preliminary experiments was relatively low, about $0.006 \mathrm{wt}$ \% (sample K241). To prove the possibility of optical damage inhibition in more heavily doped samples we check now the divergence of the laser beam transmitted through $1 \mathrm{~mm}$ thick sample from PPLN that contains ten times larger amount of iron, about 0.06wt.\% (sample K243).

The samples are cut in such a way that the polished input/output faces are parallel to the domain walls. A remarkable feature of the samples from bulk PPLN is that they consist of two periodically poled parts separated by a central $2 \mathrm{~mm}$ single-domain area (near the boule axis). This is useful for comparison of the data, obtained for PPLN, with those related to the single-domain part: all the parameters of every sample except its domain structure are the same.
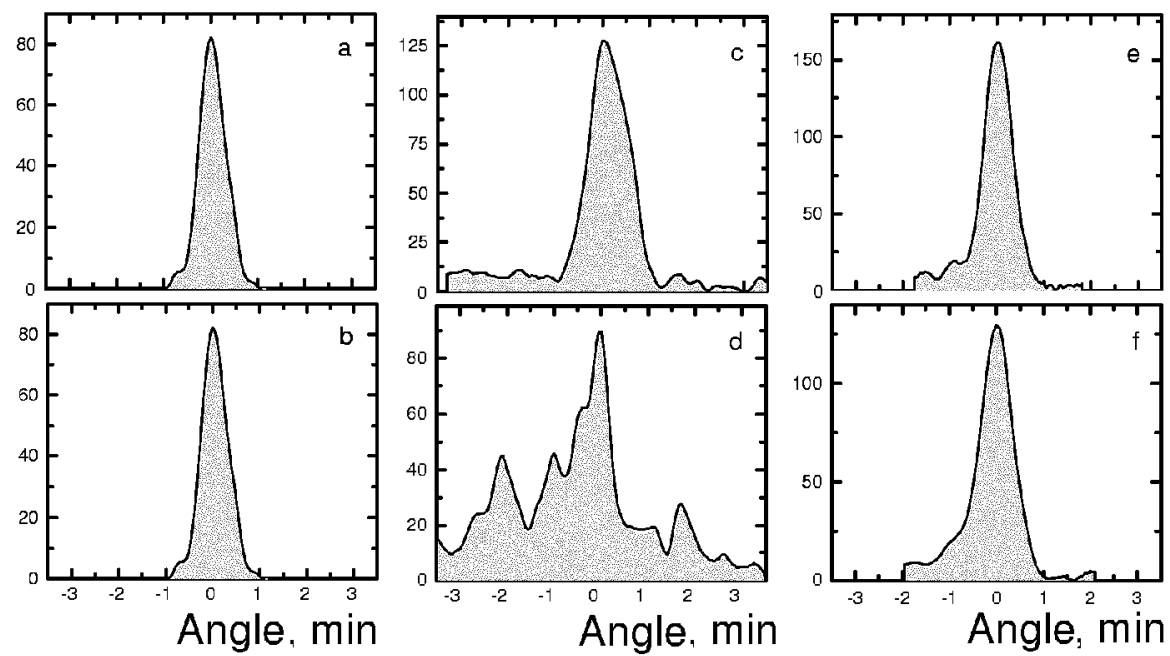

Fig. 3. Far field intensity distribution for frequency-doubled $\mathrm{Nd}^{3+}$ :YAG laser (a), (b), for the same laser beam transmitted through the single domain area of the sample (c), (d) and transmitted through PPLN area (e), (f). The light wave inside the sample is ordinary for (c), (e) and extraordinary for (d), (f). PPLN sample K243, $\ell=1 \mathrm{~mm}$.

Figure 3 represents the far-field intensity distribution of the output beam from the frequency doubled diode pumped $\mathrm{Nd}^{3+}: \mathrm{YAG}$ laser (a), (b), of the same beam transmitted through the single-domain part of the sample (c), (d) and transmitted through PPLN part of the sample (e), (f). The upper row of the graphs (a), (c), (e) shows the data for ordinarily polarized light inside the sample while the lower row (b), (d), (f) gives the data for extraordinarily polarized light. The laser beam waist at the input face of the sample is about $1.2 \mathrm{~mm}$.

As one can see, the divergence of the beam transmitted through the PPLN part of the sample (e), (f) is much closer to the divergence of the incident laser beam (a), (b) as compared to the divergence of strongly deteriorated wave trans- 
mitted through the single-domain part of the sample (c), (d). The effect is less pronounced (but nevertheless obviously present) for ordinarily polarized light because the relevant electrooptic coefficient for ordinary wave $r_{133}=8 \mathrm{pm} / \mathrm{V}$ is 3.5 times smaller than that for extraordinary wave $r_{333}=30 \mathrm{pm} / \mathrm{V}$ [12].

\section{Recording of 3D phase gratings with orthogonally polarized light waves}

Successful recording of high efficiency 3D phase gratings in PPLN:Y:Fe with two identically polarized light waves (extraordinary waves of the crystal) have been already reported in [5]. One particular feature of the photovoltaic charge transport consists in possibility of photovoltaic current excitation by two waves that are polarized orthogonally, one ordinary and other extraordinary $[8,13]$. These two waves do not produce the spatial variation of intensity but they give rise to spatial modulation of polarization of the total light field. Some components of photovoltaic tensor for crystals of $3 m$ symmetry $\left(\mathrm{LiNbO}_{3}\right)$, like, e.g., $\beta_{131}^{a}$ and $\beta_{232}^{a}$ just define the currents that depend on polarization of incident field; these currents change the propagation direction to opposite one whenever counterclockwise circular polarization is transformed into clockwise (so-called "circular photovoltaic currents"). This makes possible the recording of the space-charge gratings by two orthogonally polarized coherent light waves [13]. The anisotropic diffraction from these gratings is allowed because of non-vanishing components $r_{313}$ and $r_{323}$ in electrooptic tensor of lithium niobate. In our paper written and presented at Waseda Symposium in 1997 [14] we pointed out that circular photovoltaic currents can be efficiently used for grating recording in PPLN as well.

In the experiment the light beams from the frequency doubled diode pumped $\mathrm{Nd}^{3+}$ :YAG laser are used for grating recording in $1 \mathrm{~mm}$ thick sample of PPLN

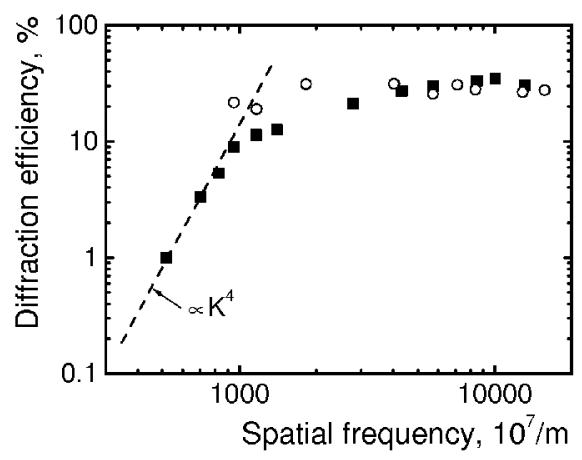

Fig. 4. Measured dependence of the diffraction efficiency versus grating spatial frequency for polarization anisotropic grating recording. Filled squares correspond to PPLN part while open dots correspond to single domain area. PPLN sample K243, $\ell=1 \mathrm{~mm}$. 
K243. Figure 4 shows the dependence of the diffraction efficiency of the recorded grating on spatial frequency. It is worthwhile to underline rather high diffraction efficiency (the largest measured value for optimized intensity ratio of the recording waves reaches $60 \%$ ) and characteristic $K^{4}$ drop down of the efficiency at low spatial frequencies that reflects $K^{2}$ dependence of the refractive index change according to $\mathrm{Eq} .(5)$.

\section{Coherent optical oscillation}

Photorefractive crystal pumped by two counterpropagating waves may serve as a phase conjugate mirror (see, e.g., [15]). If the reflectivity of such a phase conjugate mirror becomes larger than $100 \%$ (amplified phase conjugate reflectivity) it can form together with the ordinary high reflecting mirror a cavity and coherent oscillation may occur [16]. Two possible geometries used in this study to get the self-oscillation in "semilinear" cavity [15] are depicted in Fig. 5. This particular type of coherent oscillator attracts special interest of researchers because of its ability to compensate for intracavity phase distortions.

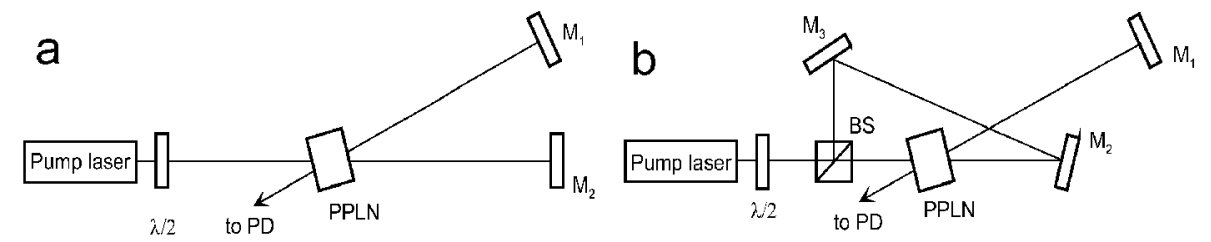

Fig. 5. Experimental geometry to study coherent oscillation in PPLN, with retroreflected pump beam (a) and with two independent pump beams (b). $M$ are the mirrors, $\lambda / 2$ are the phase retarders, $B S$ is the beam splitter, and $P D$ (not shown in this figure) are the photodetectors.

The coherent oscillation with bulk homogeneously poled $\mathrm{LiNbO}_{3}: \mathrm{Fe}$ was achieved for the first time already long ago $[17,18]$. The angular divergence of the oscillation wave have however been orders of magnitude larger than the diffraction limit, on the order of few degree of arc [18] because of the strong optical damage induced in the sample by the pump and oscillation waves.

We repeated now these experiments taking PPLN:Y:Fe instead of bulk iron doped lithium niobate. The same $\operatorname{Ar}^{+}$-laser $(\lambda=0.488 \mu \mathrm{m})$ as in Ref. [18] is used to form the pump waves. Two possible linear polarizations of the pump wave have been used (extraordinary and ordinary waves of the crystal), as also two different orientations of the sample ferroelectric axis (in plane of pump wave and oscillation wave and normal to this plane). In this way we were able to test the photovoltaic currents that are due to all strongest components of photovoltaic tensor in iron-doped $\mathrm{LiNbO}_{3}: \mathrm{Fe}, \beta_{333}, \beta_{322}$, and $\beta_{232}^{a}$. The self-oscillation has been 
obtained with extraordinary pump waves and grating vector $\boldsymbol{K} \| \boldsymbol{O Z}\left(\beta_{333}\right)$, with ordinary pump waves and grating vector $\boldsymbol{K} \| \boldsymbol{O Z}\left(\beta_{322}\right)$ and with ordinary pump waves and grating vector $\boldsymbol{K} \| \boldsymbol{O Y}\left(\beta_{232}\right)$. The polarization of oscillation wave was extraordinary, ordinary, and extraordinary for these three cases, respectively. It was not possible to achieve the ordinary self-oscillation with extraordinary pump because of unfavourable direction of intensity coupling between two crystal eigenmodes [19] similarly to the bulk homogeneously poled iron-doped lithium niobate.

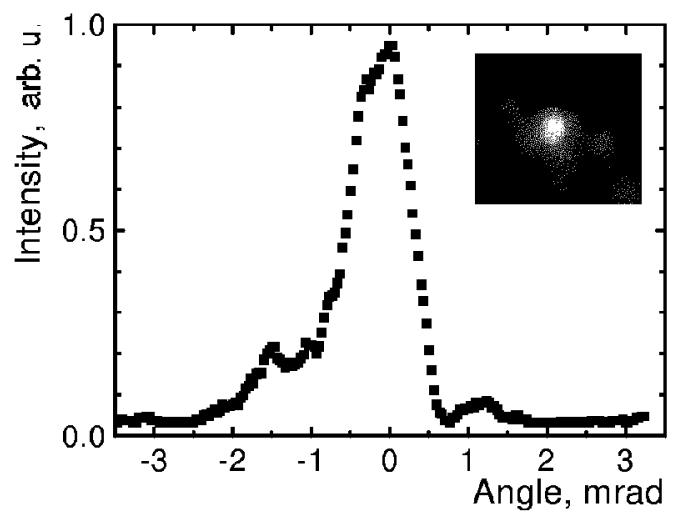

Fig. 6. Far-field pattern recorded with CCD camera (insert) and far field intensity distribution of the oscillation wave. PPLN sample K243, $\ell=1 \mathrm{~mm}$, the length of semilinear cavity is $5 \mathrm{~cm}$, the cavity axis makes angle $20^{\circ}$ to the pump propagation.

First of all, we check the angular divergence of a new generated oscillation beam. Figure 6 shows the particular result for the third from three configurations mentioned above (ordinary pump wave, extraordinary oscillation wave). The far field intensity distribution is recorded with the CCD camera (Fig. 6a) and the measurements in horisontal plane (Fig. 6b) are presented. One can see that the HWHM divergence is about one mrad. It is still larger than the diffraction limit for $1.2 \mathrm{~mm}$ beam waist of a laser emitting at $\lambda=0.488 \mu \mathrm{m}$ but it is roughly two orders of magnitude smaller than previously published data for homogeneously poled bulk $\mathrm{LiNbO}_{3}: \mathrm{Fe}$ [18]. With such a quality of output wave front this semilinear coherent oscillator can be used for compensation of intracavity phase distortions.

Figure 7 represents typical dynamics of onset of coherent oscillation. A virgin crystal (with all previously recorded gratings erased by crystal heating to $200^{\circ} \mathrm{C}$ during 1 hour) is illuminated by the pump waves starting from $t=0$. By tilting the linear polarization of the pump wave to the angle $\varphi$ it is possible to reduce the effective coupling strength of the sample as $\gamma \ell=(\gamma \ell)_{\max } \cos ^{2} \varphi$. Different curves in Fig. 7 correspond to different coupling strengths controlled in such a way.

During relatively long time the light intensity scattered into direction of future oscillation wave (i.e., along the cavity axis) remains relatively small until 

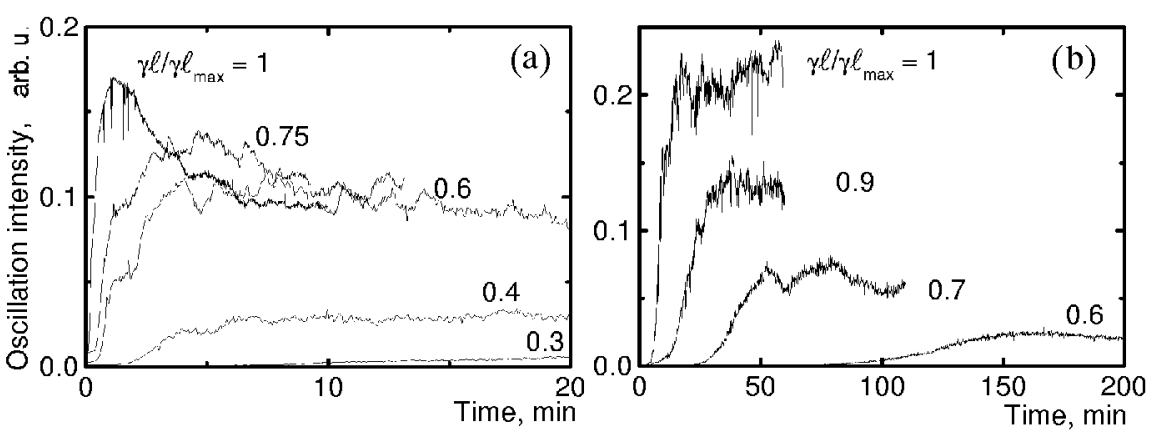

Fig. 7. Temporal variation of oscillation intensity for extraordinary pump and extraordinary oscillation wave (a) and for ordinary pump and ordinary oscillation wave (b). The numbers near the curves indicate how strongly the coupling strength is reduced compared to its ultimate value when the linear polarization of the pump wave is rotated. PPLN sample K241, $\ell=4 \mathrm{~mm}$. The length of the semilinear cavity is $10 \mathrm{~cm}$, the cavity axis makes angle $13^{\circ}$ to pump propagation.

the nonlinear growth of intensity occurs. The smaller is the coupling strength the longer becomes the delay time which is necessary to achieve the self-oscillation. This phenomenon is known for coherent oscillators of various types (see, e.g., [20]) as also for usual lasers (see, e.g., [21]), it has a close analogy with critical slowing down for the second-order phase transitions.

Figure 8 shows the coupling strength dependences of saturated oscillation intensity (a) and oscillation switch-on time (b) for two first oscillator geometries (extraordinary pump - extraordinary oscillation wave and ordinary pump - ordinary oscillation wave). In both cases the threshold coupling strength can be determined easily which is important for evaluation of photovoltaic constant, described in the next section of this article.

\section{Evaluation of photovoltaic parameters from the threshold conditions of oscillation}

From the dependences shown in Fig. 8 one can imagine how large is the ultimate coupling strength ensured by the sample as compared to the threshold coupling strength that is necessary to switch on the oscillation. To get this ratio we do not need to know the exact values, the normalized values like presented in Fig. 8 are sufficient. One can see that for extraordinary pump wave the ultimate $\gamma \ell$ is 4 times larger than the threshold value $\gamma \ell_{\mathrm{th}}$. In fact, for optimized pump ratio (using the experimental geometry shown in Fig. 5b) we were able to reach the self-oscillation even for coupling strength ten times smaller than the ultimate value ensured by $4 \mathrm{~mm}$ thick crystal. If now we can evaluate the threshold coupling strength we will obtain also the ultimate coupling strength for our sample which will allow for evaluation of photovoltaic constant. 

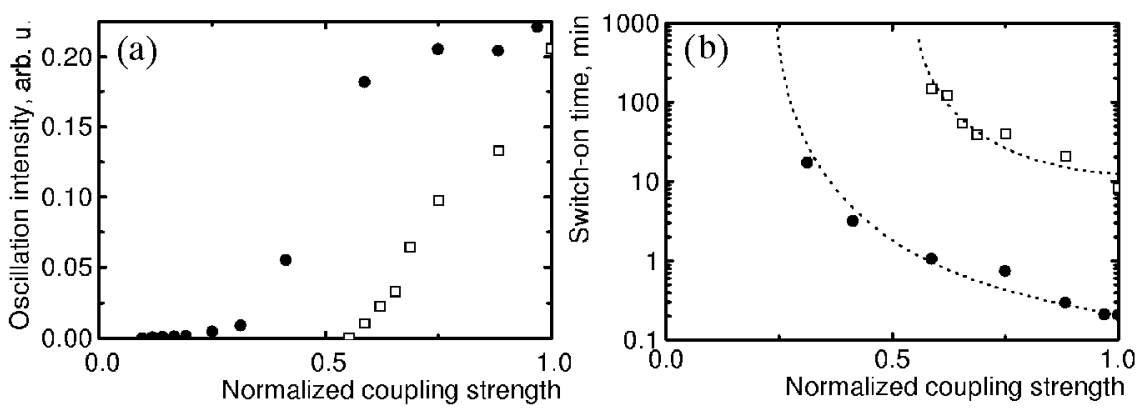

Fig. 8. Measured coupling strength dependence of oscillation intensity (a) and oscillation switch-on time (b). Filled dots correspond to extraordinary pump and extraordinary oscillation waves while open squares correspond to ordinary pump and ordinary oscillation wave. PPLN sample K241, $\ell=4 \mathrm{~mm}$. The dashed lines are shown to guide the eye.

The amplitude condition of oscillation imposes that the intensity of oscillation wave should be the same after one round trip of the cavity, i.e.,

$$
R \cdot R_{\mathrm{pc}}=1
$$

where $R$ is the (intensity) reflectivity of the ordinary mirror, while $R_{\mathrm{pc}}$ is the (intensity) phase conjugate reflectivity of the nonlinear crystal. We neglect, for the sake of simplicity, all other cavity losses, like Fresnel reflections from the crystal, crystal absorption, etc. Our estimate therefore will give only the lower limit for the threshold coupling strength.

The lowest calculated value of the threshold coupling strength can be found from simple theory of backward-wave four-wave mixing [15]. For purely local nonlinear response (which is well justified for photovoltaic charge redistribution because of $\beta_{333}$ component of the current) and equal intensities of the pump waves to optimize phase conjugate reflectivity we get

$$
R_{\mathrm{pc}}=\tanh ^{2}\left(\frac{\gamma \ell}{2}\right) \text {. }
$$

From Eq. (6) we get therefore for $R=1$ the threshold coupling strength equal to

$$
\gamma \ell_{\mathrm{th}}=\pi / 2 \text {. }
$$

Thus we know that our $4 \mathrm{~mm}$ thick PPLN sample ensures at least $\gamma \ell=5 \pi$ for interaction of extraordinary waves with $\beta_{333}$ involved.

Taking into account the definition of the coupling strength for photovoltaic photorefractive crystals [4]

$$
\gamma \ell=\frac{\pi r_{\mathrm{eff}} n^{3}}{\lambda} E_{\mathrm{pv}}
$$

we can estimate now the effective photovoltaic field. With $r_{333} \approx 30 \mathrm{pm} / \mathrm{V}$ and $n \approx 2.2$ (we neglect birefringence for simplicity) one can get 


$$
E_{\mathrm{pv}} \approx 40 \mathrm{kV} / \mathrm{cm} \text {. }
$$

This value seems to be not surprising for iron doped lithium niobate. It should be underlined however that it is obtained for the crystal with large amount of other dopant, yttrium with the density $\approx 0.74 \mathrm{wt}$.\% that is much higher than the density of photorefractive impurity, $\approx 0.006-0.06 \mathrm{wt} . \%$. In such a way our study shows that, fortunately, even relatively large amount of yttrium cannot fully suppress photovoltaic and photorefractive effects in iron doped $\mathrm{LiNbO}_{3}$. Note that $E_{\mathrm{pv}}$ given by Eq. (10) is obviously underestimated because the losses of the cavity were not taken into account in full extent.

\section{Stabilization of ultimate grating efficiency with the active feedback}

The data presented in previous section show that inherent coupling strength for $4 \mathrm{~mm}$ thick PPLN sample may reach and overpass $5 \pi$. This value is much higher than that which is necessary to get $100 \%$ diffraction efficiency when recording a volume phase grating by two light beams with equal intensities $(\gamma \ell=\pi)$. Even the $1 \mathrm{~mm}$ thick sample provides the overall phase modulation which is larger than that necessary to get $100 \%$ grating efficiency. The use of two beams of unequal intensities reduces the diffraction efficiency immediately because of intensity dependent nonlinear phase modulation and subsequent bent of the grating fringes [22].

To overcome this difficulties we use the active feedback grating recording proposed and implemented for the first time in [23-25]. The input phase of one of two recording beams is controlled by an electronic feedback system shown in Fig. 9 that includes a lock-in amplifier. A special signal generator produces a very small (with the amplitude $\psi \ll \pi$ ) and very fast (at frequency $\omega \gg 1 / \tau_{\mathrm{di}}, \tau_{\mathrm{di}}$ being the dielectric relaxation time) periodic phase modulation, $\Delta \varphi_{\mathrm{s}} \propto \sin \omega t$ of the input recording beam. Such a modulation does not affect the buildup of the grating and serves only for controlling the feedback loop. Because of the coupling through the recorded grating both transmitted beams possess, in addition to a constant part, also weak components oscillating as $\sin \omega t$ and $\cos 2 \omega t$. Using the amplitude $I_{2 \omega}$ as an error signal in the electronic feedback loop and keeping positive the amplitude $I_{\omega}$, one can ensure the desirable active feedback.

Sending the interested reader for details to the original publications [23-26] we will limit ourselves here only by qualitative description of feedback operation. According to the theory the amplitudes of the $I_{\omega}$ and $I_{2 \omega}$ signals are given by the expressions

$$
\begin{aligned}
& I_{\omega}=2\left|R_{0} S_{0}\right| \psi \sin \varphi \sqrt{\eta(1-\eta)} \\
& I_{2 \omega}=0.5\left|R_{0} S_{0}\right| \psi^{2} \cos \varphi \sqrt{\eta(1-\eta)},
\end{aligned}
$$

where $R_{0}$ and $S_{0}$ are the amplitudes of the input recording beams, $\psi$ is an amplitude of the initial (high frequency) phase modulation, $\varphi$ is the phase difference between the transmitted and diffracted components propagating in the same direction and $\eta$ is the diffraction efficiency. 


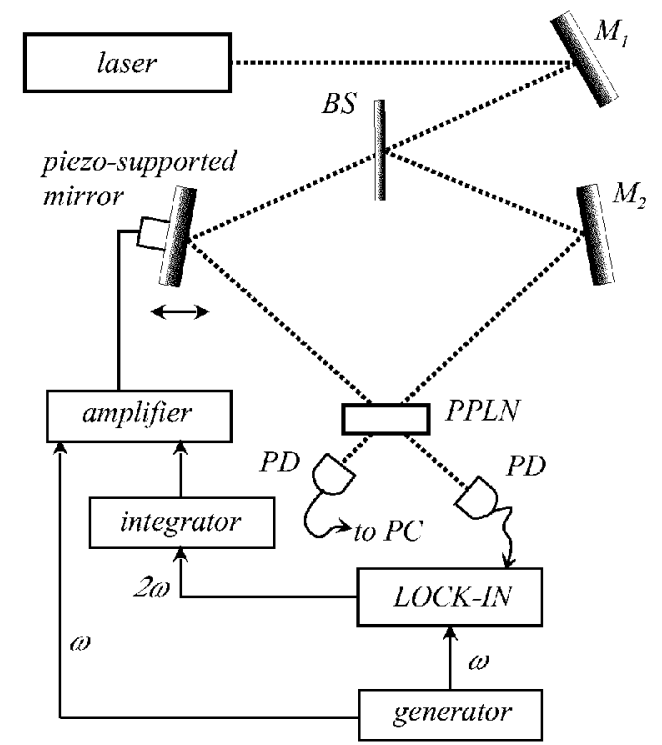

Fig. 9. Schematic representation of the feedback assisted grating recording. $M$ are the mirrors, $P D$ are the photodetectors, $B S$ is the beam splitter, $P C$ is a computer. The angle between recording waves is $12^{\circ}$.

It is obvious from Eq. (12) that $I_{2} w$ will become identically zero either for $\phi=\pi / 2$ or for $\eta=1$ or for trivial situation with no grating at all, $\eta=0$, independently of what are the values of other factors in this equation. Thus one can expect that active feedback will drive whole recording system to the smallest $I_{2 \omega}$, i.e., to the largest possible $\eta$.

A theoretical analysis of the active stabilization performed recently in [26] described the details of behaviour of active feedback system when grating efficiency reaches already $100 \%$ value. It has been shown that due to the inertia of the feedback loop the different, nearly periodic phase modulations are introduced to the recording beam, which keeps the grating very close to its $100 \%$ efficiency (with deviations much less than $1 \%$ ).

It should be mentioned that in addition to $100 \%$ diffraction efficiency the active feedback ensures the reversal of intensity ratio at the crystal output, i.e., provides an efficient beam coupling for the input beams with unequal intensities.

Figure 10 shows the temporal dynamics of active feedback operation. The temporal variation of the output beam intensities is shown in Fig. 10a. One can see that the intensity ratio in saturation becomes nearly inverse with respect to the initial one $2: 1$. Both beams are partially depleted in saturation due to the light induced scattering. The voltage generated by the feedback loop and applied to piezodrive to change the phase of one of the input waves is shown in Fig. 10b. And finally, Fig. 10c shows the dynamics of the first harmonic signal $I_{\omega}$. (We 

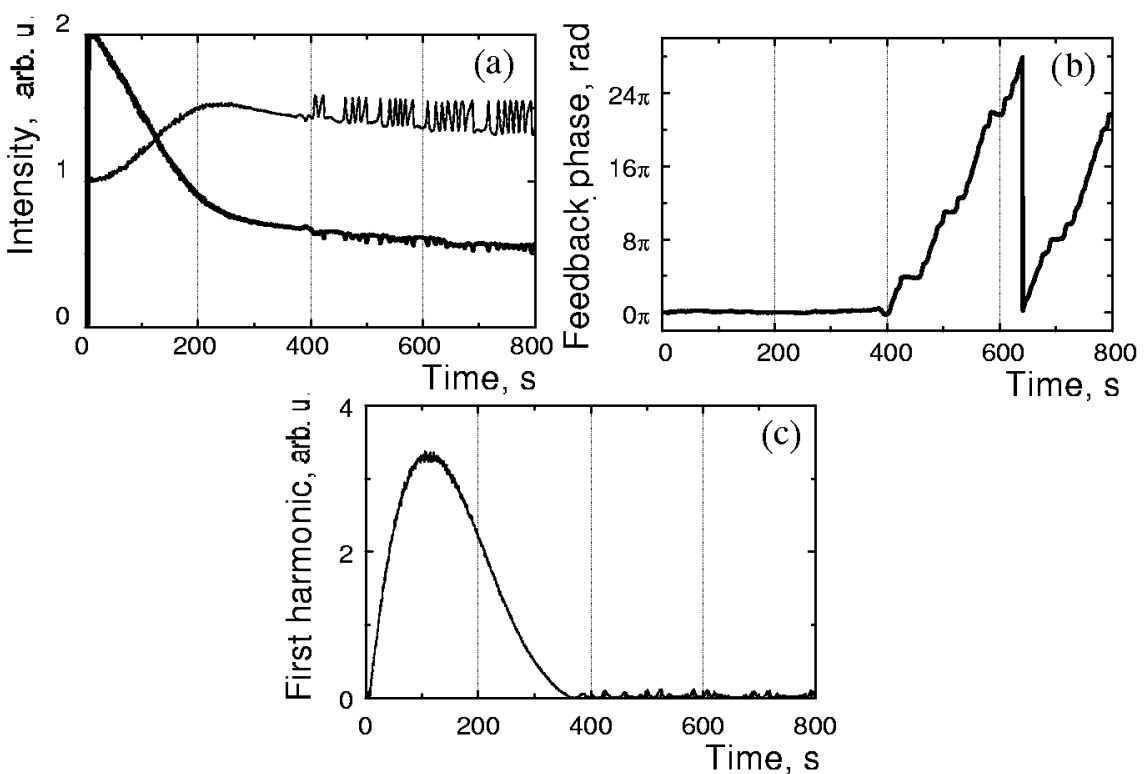

Fig. 10. Temporal variation of the output intensities of the recording beams (a), of the voltage driving piezomirror in the recording beam (b), and of the $I_{\omega}$ modulation amplitude. Note the dramatic change in dynamics for $t=400 \mathrm{~s}$, when the diffraction efficiency reaches $100 \%$ value. PPLN sample K241, $\ell=4 \mathrm{~mm}$.

choose $I_{\omega}$ to be measured experimentally because it is much larger than $I_{2 \omega}$ but has the similar dependence on the diffraction efficiency.)

One can see that approximately at $t \approx 400 \mathrm{~s}$ the amplitude of $I_{\omega}$ drops to the noise level and at the same time the feedback controlled mirror starts to introduce rather strong nearly regular phase variations in the recording wave. From visual inspection we see the appearance of the black spot in the transmitted readout beam when the other recording beam is temporarily stopped. This confirms that the diffraction efficiency is close to its ultimate $100 \%$ value and total intensity of the readout beam is diffracted in the direction of the second recording beam.

By using Eq. (11) we reconstruct from the data of Fig. 10c the temporal dynamics of the grating efficiency, which is shown in Fig. 11. Figure 11b shows the detailed behaviour of $\eta$ near saturated value, it is clear that deviation from $100 \%$ efficiency is really smaller than $10^{-3}$. It should be underlined that the data of Fig. 11 are not measured but calculated, the real diffraction efficiency is smaller than $100 \%$ because of the Fresnel losses from the sample face reflections, because of crystal absorption, and because of initial and light induced scattering. What the active feedback provides us with is the stabilization of the largest possible diffraction efficiency that corresponds roughly to the first maximum of the known Kogelnik equation describing diffraction efficiency of the symmetric volume phase grating [27]. 

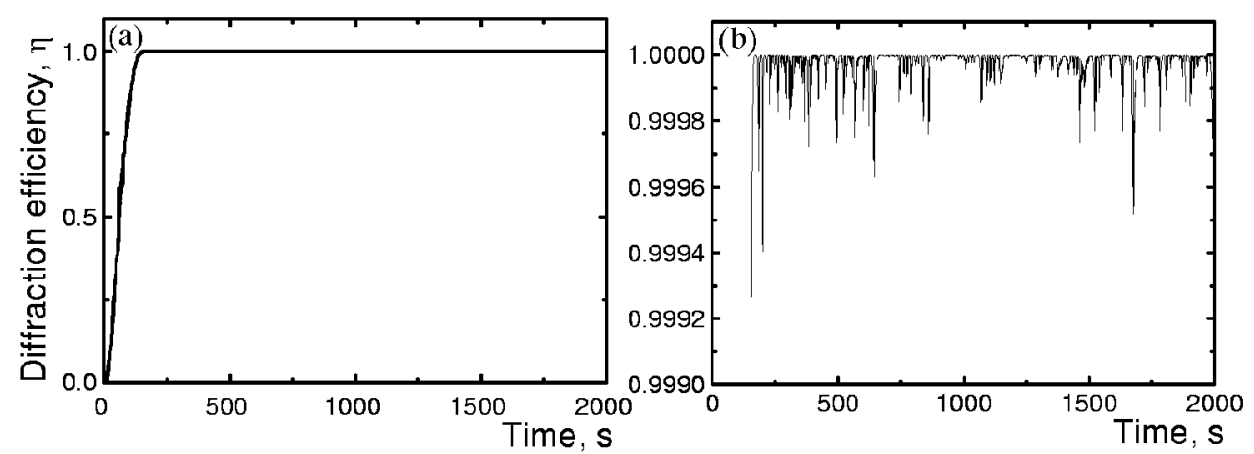

Fig. 11. Temporal variation of the diffraction efficiency calculated from the data of Fig. 10c. Full range variation of $\eta$ are shown in (a) while (b) represents the detailed behaviour of $\eta$ in saturation.

One of the issues of the active feedback recording consists in "unbending" of the grating otherwise tilted when the recording waves have unequal intensities. To check this effect directly we measure and compare the angular selectivity curves for grating recording in the same sample with feedback on and off providing the initial intensity ratio is equal to 1:10. The results are shown in Fig. 12. The normalized diffraction efficiency is plotted as a function of angular deviation of the readout beam from its position during recording. To measure these dependences the intensity of the readout beam was deliberately reduced in order to prevent from grating erasure during the measurements.

(a)

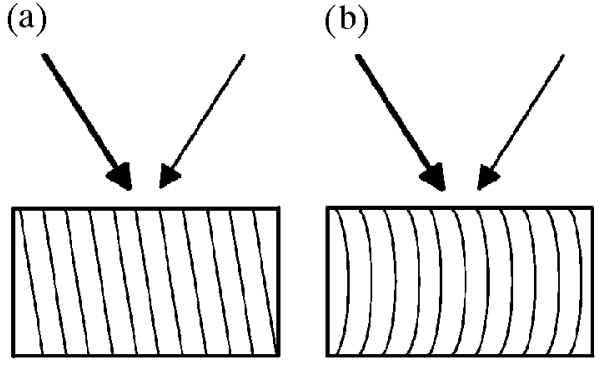

(b)

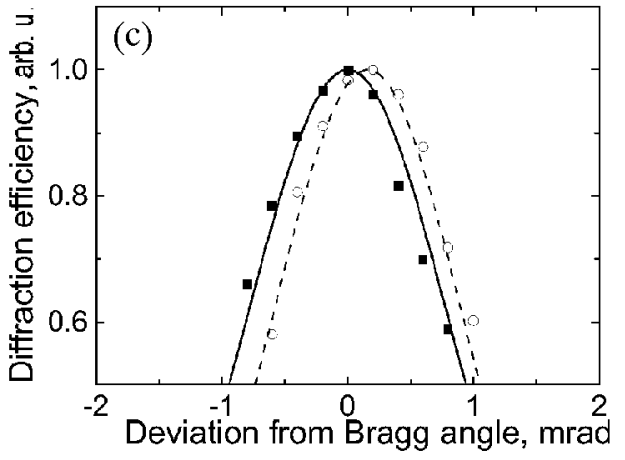

Fig. 12. Schematic representation of the grating structure with no feedback (a) corresponding to dashed angular selectivity curve in (c) and with feedback assisted recording (b) corresponding to solid angular selectivity curve in (c).

It is obvious that for recording with no feedback the largest efficiency corresponds to a certain inclination of the readout beam from its initial position, i.e., the recorded grating appear to be tilted. For the feedback assisted recording, however, we get nearly symmetric angular selectivity curve with the maximum exactly at the recording beam position. 


\section{Parametric photorefractive scattering}

The first observation and experimental study of parametric photorefractive scattering in periodically poled $\mathrm{LiNbO}_{3}: \mathrm{Y}: \mathrm{Fe}$ crystals has been reported in [6]. The detection of pronounced parametric processes is a direct proof of the efficiency of this engineered nonlinear material. Furthermore, the presence of the periodic domain structure leads to a new set of phase-matched parametric processes and allows for the observation of many new scattering processes in bulk PPLN:Y:Fe. Development of these new types of scattering is possible not only for identically but also for orthogonally polarized pump beams.

The practical interest to parametric mixing of co-propagating waves is related to very high gain that can be achieved (gain factor as large as hundreds reciprocal centimeters) as in traditional (known for bulk homogeneously poled crystals) as also in new (involving domain lattice vector and therefore typical only of PPLN) nonlinear mixing processes.

\section{Conclusions}

The doped periodically poled lithium niobate (PPLN:Y:Fe) possesses nearly the same photorefractive sensitivity for grating recording, as that of the bulk homogeneously poled crystal. It allows for efficient grating recording both with identically polarized light waves or with orthogonally polarized crystal eigenwaves.

Co-doped PPLN:Y:Fe exhibits pronounced bulk photovoltaic effect, strong enough to ensure high photorefractive sensitivity for grating recording. This means that even large amount of yttrium in the samples does not reduce dramatically the photorefraction related to iron photorefractive centres.

Highly efficient frequency degenerate wave mixing is possible with PPLN:Y:Fe, in classical backward-wave geometry (phase conjugation, various photorefractive oscillators) as well as in forward-wave geometry (parametric amplification of co-propagating coherent seed).

The presence of a domain lattice gives rise to a large number of new phase-matched parametric processes that ensure rather high parametric gain.

\section{Acknowledgments}

We are grateful to Dr. I. Naumova for PPLN samples, to F. Agulló-Lopez, L. Hesselink, and P. Kazanski for stimulating discussions. Partial financial support from INTAS (grant 97-31275) and from CRDF (grant UP2-2122) is gratefully acknowledged.

\section{References}

[1] J.P. van der Ziel, N. Bloembergen, Bull. Am. Phys. Soc. 8, 380 (1963).

[2] R.L. Byer, J. Nonlinear Opt. Phys. Mater. 6, 549 (1997). 
[3] G. Rosenman, A. Skliar, A. Arie, Ferroelectric Review 1, 263 (1999).

[4] B. Sturman, Maria Aguilar, F. Agulló-Lopez, V. Pruneri, P.G. Kazansky, J. Opt. Soc. Am. B 14, 2641 (1997).

[5] S. Odoulov, T. Tarabrova, A. Shumelyuk, I.I. Naumova, T.O. Chaplina, Phys. Rev. Lett. 84, 3294 (2000).

[6] M. Goulkov, S. Odoulov, I. Naumova, F. Agulló-Lopez, G. Calvo, E. Podivilov, B. Sturman, V. Pruneri, Phys. Rev. Lett. 86, 4021 (2001).

[7] Photorefractive Materials and Their Applications $I$ and II, Eds. P. Guenter, J.-P. Huignard, Springer Topics in Applied Physics, Vol. 61 and 62, Springer, Berlin 1988, 1989.

[8] B.I. Sturman, V.M. Fridkin, The Photovovoltaic and Photorefractive Effects in Noncentrocymmetric Materials, Gordon and Breach, Philadelphia 1992.

[9] L. Solymar, D. J. Webb, A. Grunnet-Jepsen, The Physics and Applications of Photorefractive Materials, Clarendon Press, Oxford 1996.

[10] M. Taya, M.C. Bashew, M.M. Fejer, Opt. Lett. 21, 857 (1996).

[11] I.I. Naumova, N.F. Evlanova, O.A. Gliko, S.V. Lavrischev, J. Cryst. Growth 181, 160 (1997).

[12] S.H. Wemple, M. Di Domenico, J. Camlibel, J. Chem. Solid 29, 1797 (1968).

[13] A. Novikov, S. Odoulov, O. Oleinik, B. Sturman, Ferroelectrics 74, 295 (1987).

[14] S. Odoulov, B. Sturman, in: Progress in Photorefractive Nonlinear Optics, Proc. Waseda Symp., Ed. K. Kuroda, Francis and Taylor, Tokyo 2002, in press.

[15] M. Cronin-Golomb, B. Fischer, J.O. White, A. Yariv, IEEE J. Quantum Electron. QE-20, 12 (1984).

[16] R. Hellwarth, J. Feinberg, Opt. Lett. 5, 519 (1980).

[17] S. Odoulov, M. Soskin, JETP Lett. 37, 289 (1983).

[18] S. Odoulov, Sov. J. Quantum Electron. 14, 530 (1984).

[19] S. Odoulov, Ferroelectrics 91, 213 (1988).

[20] M. Goul'kov, O. Shinkarenko, S. Odoulov, E. Kraetzig, R. Pankrath, Appl. Phys. B 72, 187 (2001).

[21] R. Loudon, M. Harris, T. Sheppard, J. Vaughan, Phys. Rev. A 48, 681 (1993).

[22] N. Kukhtarev, V. Markov, S. Odoulov, M. Soskin, V. Vinetskii, Ferroelectrics 22, 949 (1979).

[23] A.A. Freschi, J. Frejlich, J. Opt. Soc. Am. B 11, 1837 (1994).

[24] P.M. Garcia, A.A. Freschi, J. Frejlich, E. Kraetzig, Appl. Phys. B 63, 207 (1996).

[25] A.A. Freschi, P.M. Garcia, I. Rasnik, J. Frejlich, K. Buse, Opt. Lett. 21, 152 (1996).

[26] E.V. Podivilov, B.I. Sturman, S.G. Odoulov, S. Pavlyuk, K.V. Shcherbin, V.Ya. Gayvoronsky, K.H. Ringhofer, V.P. Kamenov, Phys. Rev. A 63, 053805 (2001).

[27] H. Kogelnik, Bell Syst. Techn. J. 48, 2909 (1969) 